\title{
Atorvastatin promotes AMPK signaling to protect against high fat diet-induced non-alcoholic fatty liver in golden hamsters
}

\author{
BIN ZHANG $^{1-4^{*}}$, CHENYANG ZHANG ${ }^{1-4^{*}}$, XUELIAN ZHANG ${ }^{1-4}$, NANNAN LI $^{1}$, \\ ZHENGQI DONG ${ }^{1}$, GUIBO SUN ${ }^{1-4}$ and XIAOBO SUN ${ }^{1-4}$ \\ ${ }^{1}$ Institute of Medicinal Plant Development, Peking Union Medical College and Chinese Academy of Medical Sciences; \\ ${ }^{2}$ Key Laboratory of Bioactive Substances and Resources Utilization of Chinese Herbal Medicine, \\ Ministry of Education; ${ }^{3}$ Beijing Key Laboratory of Innovative Drug Discovery of Traditional Chinese Medicine \\ (Natural Medicine) and Translational Medicine; ${ }^{4}$ Key Laboratory of Efficacy Evaluation of Chinese Medicine against \\ Glycolipid Metabolism Disorder Disease, State Administration of Traditional Chinese Medicine, Beijing 100193, P.R. China
}

Received December 21, 2018; Accepted December 11, 2019

DOI: $10.3892 /$ etm.2020.8465

\begin{abstract}
Non-alcoholic fatty liver disease (NAFLD) is characterized by diffuse fatty acid degeneration and excess fat accumulation in the liver. Notably, the currently available medications used to treat NAFLD remain limited. The aim of the present study was to investigate the protective role of atorvastatin (Ato) against NAFLD in golden hamsters fed a high fat diet (HFD) and in HepG2 cells treated with palmitate, and identify the underlying molecular mechanism. Ato ( $3 \mathrm{mg} / \mathrm{kg}$ ) was administered orally every day for 8 weeks to the hamsters during HFD administration. Hamsters in the model group developed hepatic steatosis with high serum levels of triglyceride, cholesterol, insulin and C-reactive protein, which were effectively reduced by treatment with Ato. Additionally, the relative liver weight of hamsters treated with Ato was markedly lower compared with that of the model group. Hematoxylin and eosin, and oil red $\mathrm{O}$ staining indicated that the livers of the animals in the model group exhibited large and numerous lipid droplets, which were markedly decreased after Ato treatment. Western blot analysis indicated that Ato inhibited fat accumulation in the liver through the AMP-activated protein kinase (AMPK)-dependent activation of peroxisome proliferator activated receptor $\alpha$ (PPAR $\alpha$ ), peroxisome proliferator-activated receptor- $\gamma$ coactivator $1 \alpha$
\end{abstract}

Correspondence to: Professor Guibo Sun or Professor Xiaobo Sun, Institute of Medicinal Plant Development, Peking Union Medical College and Chinese Academy of Medical Sciences, 151 Malianwa North Road, Haidian, Beijing 100193, P.R. China

E-mail: sunguibo@126.com

E-mail: sun_xiaobo163@163.com

${ }^{*}$ Contributed equally

Key words: atorvastatin, non-alcoholic fatty liver, lipolysis, lipid accumulation, AMP-activated protein kinase and their target genes. Furthermore, in vitro, Ato inhibited PA-induced lipid accumulation in HepG2 cells. This inhibitory effect was attenuated following Compound $\mathrm{C}$ treatment, indicating that AMPK may be a potential target of Ato. In conclusion, the increase in AMPK-mediated PPAR $\alpha$ and its target genes may represent a novel molecular mechanism by which Ato prevents NAFLD.

\section{Introduction}

The prevalence of non-alcoholic fatty liver disease (NAFLD) is increasing worldwide, and $\sim 25 \%$ of adults worldwide suffer from this disease (1). It is estimated that in developed countries $\sim 34 \%$ of children who are obese may have NAFLD $(2,3)$. Due to the lack of symptoms, NAFLD is challenging to diagnose, and the progression of this disease is unpredictable. Type 2 diabetes mellitus (T2DM) and cardiovascular diseases are reported to be associated with NAFLD, and are the leading causes of mortality in patients with NAFLD $(4,5)$. Basic and clinical studies have shown that NAFLD is detrimental to the health of patients $(6,7)$. Therefore, appropriate therapeutic strategies and drugs are required for the treatment of NAFLD.

NAFLD is characterized by diffuse fatty acid (FA) degeneration and excessive fat accumulation in the liver. Therefore, NAFLD is often accompanied by increased central adiposity and triglycerides (TGs), hyperlipidemia and low levels of high-density lipoprotein (HDL), and this disease is now well recognized as a type of metabolic syndrome $(8,9)$. The mechanisms resulting in the progression of hepatic steatosis are complex, and include increased de novo FA generation (lipogenesis), decreased $\beta$-oxidation and enhanced non-esterified FA release from adipose tissue (lipolysis) $(10,11)$. Caloric restriction and exercise can improve NAFLD (12), but changing lifestyle can be challenging for most patients with NAFLD. To the best of our knowledge, with the exception of diet and lifestyle modifications, no effective treatments for NAFLD are currently available (13). Therefore, the identification of effective drugs and investigation of their protective mechanism in the control of lipid levels is required for the treatment of NAFLD. 
Atorvastatin (Ato), a lipid-decreasing agent, is the most commonly prescribed statin drug worldwide (14), and is used for the treatment of hypercholesterolemia or mixed dyslipidemia. Mechanistically, Ato exerts its protective roles by competitively inhibiting 3-hydroxy-3-methyl-glutaryl-coenzyme A (HMG-CoA) reductase, which is known to suppress the mevalonate pathway and subsequently de novo hepatic cholesterol (CHO) synthesis (15). Due to the wide application of Ato in clinical settings, other therapeutic properties have been identified in addition to its lipid-decreasing activity, and this drug has been used in the treatment of various disorders, including endothelial dysfunction, cardiovascular disease and depression $(16,17)$. However, studies investigating the ability of Ato to prevent NAFLD are limited, and its molecular mechanisms are not fully understood (18). Therefore, it is necessary to examine the potential protective roles and underlying mechanisms of Ato in the treatment of NAFLD in order to identify evidence supporting the clinical application of this drug.

In the present study, golden hamsters were fed with a high-fat diet (HFD) to induce NAFLD. The results suggested that Ato effectively prevented the progression of NAFLD by promoting the AMP-activated protein kinase (AMPK) signaling pathway. However, following AMPK inhibition by Compound C in HepG2 cells, the inhibitory effects of Ato on lipid accumulation were suppressed. The results indicated that Ato may exhibit potential therapeutic properties for the treatment of NAFLD, at least in part, by promoting the AMPK signaling pathway and its downstream targets.

\section{Materials and methods}

Experimental animals and treatment protocols. Syrian hamsters received humane care according to the Guidelines for the Experimental Laboratory Animal Committee of the Chinese Academy of Medical Sciences and Peking Union Medical College, and the experimental protocols were approved by the Ethics Committee of the Chinese Academy of Medical Sciences and Peking Union Medical College. A total of 24 male Golden Syrian hamsters (age, 8 weeks; weight, $100 \pm 10 \mathrm{~g}$ ) were purchased from Beijing Vital River Laboratory Animal Technology Co., Ltd (Beijing, China). Hamsters were housed in a temperature-controlled environment (temperature, $22-2^{\circ} \mathrm{C}$; humidity, $55-5 \%$ ) with a 12 -h light/dark cycle and ad libitum access to food and water. To increase hepatic lipid accumulation and create the NAFLD model, 16 hamsters were fed with a HFD (20 kcal\% protein, $20 \mathrm{kcal} \%$ carbohydrate and $60 \mathrm{kcal} \%$ fat), while 8 hamsters were fed a normal diet (30 kcal\% protein, $60 \mathrm{kcal} \%$ carbohydrate and $10 \mathrm{kcal} \%$ fat) and served as a control. The diets were obtained from Beijing HFK Bioscience Co., Ltd.

After 2 weeks, 8 hamsters receiving the HFD were administered $3 \mathrm{mg} / \mathrm{kg} /$ day Ato via gavage in a volume of $1 \mathrm{mg} / \mathrm{ml}$ distilled water for 8 weeks to establish the Ato group (Fig. 1A). The other 8 hamsters receiving the HFD (model group) and the 8 hamsters in the control group received vehicle instead. The HFD and normal diets were continued during the 8-week treatment period. The daily state of the animals was recorded and their body weight was measured weekly. After 8 weeks of treatment, the animals were anesthetized using urethane
(1.2 g/kg body weight), and blood samples were subsequently extracted from the inferior vena cava for analysis. Under anesthesia, the liver tissues were removed, weighed, snap-frozen in liquid nitrogen and stored at $-80^{\circ} \mathrm{C}$. The coefficient of hepatic weight was calculated as follows: Liver weight $(\mathrm{g}) /$ body weight (100 g).

Reagents. Ato (used in the in vivo experiments) was purchased from Pfizer, Inc. CHO, TG, HDL and low-density lipoprotein (LDL) determination kits were purchased from Nanjing Jiancheng Bioengineering Institute. Ato, palmitate (PA) and Compound $\mathrm{C}$ (used in the in vitro experiments) were purchased from Sigma-Aldrich (Merck KGaA). TRIzol, PrimeScript RT Master mix and SYBR Green Master mix were purchased from Takara Bio, Inc.

Cell culture and treatment. HepG2 liver cancer cells are a classical cell model utilized in numerous studies to explore abnormalities of lipid metabolism in the liver $(17,19)$. Thus, this cell line was selected for use in in vitro experiments. The HepG2 cells (cat. no. 3111C0001CCC000035) were obtained from the Cell Resource Center, Peking Union Medical College (headquarters of the National Infrastructure of Cell Line Resource, NSTI). The identity of the cell lines was authenticated using STR profiling (CODIS; FBI). Cells were cultured in DMEM (Gibco; Thermo Fisher Scientific, Inc.) supplemented with $10 \%$ FBS (Gibco; Thermo Fisher Scientific, Inc.) and $1 \%$ penicillin/streptomycin, in a $5 \% \mathrm{CO}_{2}$ atmosphere at $37^{\circ} \mathrm{C}$. The cells were plated in six-well plates at $2 \times 10^{5}$ cells/well. The groups were as follows: i) Control group; ii) PA treated group; HepG2 cells incubated with $250 \mu \mathrm{M}$ PA at $37^{\circ} \mathrm{C}$ for $24 \mathrm{~h}$; iii) $\mathrm{PA}+$ Ato group; HepG2 cells incubated with $250 \mu \mathrm{M}$ PA and $10 \mu \mathrm{M}$ at $37^{\circ} \mathrm{C}$ for $24 \mathrm{~h}$; iv) $\mathrm{PA}+$ Ato + AMPK inhibitor group; Prior to drug treatment, HepG2 cells pre-incubated with $10 \mu \mathrm{M}$ Compound $\mathrm{C}$ at $37^{\circ} \mathrm{C}$ for $4 \mathrm{~h}$.

Biochemical analysis. Serum lipid levels, namely CHO, TG, HDL and LDL, were evaluated using the aforementioned commercial kits on an automatic biochemical analyzer (Beckman Coulter, Inc.). Insulin and C-reactive protein (CRP) levels were determined using an INS RIA kit (cat. no. HY-10069) and CRP IRMA kit (cat. no. HY-10023), respectively (Beijing Sino-UK Institute of Biological Technology).

Hematoxylin and eosin $(H \& E)$ staining. Livers were harvested from hamsters, fixed in $4 \%$ paraformaldehyde for $24 \mathrm{~h}$ (room temperature), embedded in paraffin and sectioned (thickness, $5 \mu \mathrm{m}$ ). The sections were stained with $\mathrm{H} \& \mathrm{E}$ for $10 \mathrm{~min}$ (room temperature) and dehydrated. Sections were examined under a light microscope (BX53; Olympus Corporation; magnification, x200).

Oil red staining. Oil red $\mathrm{O}$ staining was performed as previously described (20). Briefly, frozen liver sections (thickness, $5 \mu \mathrm{m}$ ) or HepG2 cells were stained with oil red $\mathrm{O}$ working solutions for $30 \mathrm{~min}$, washed with $60 \%$ isopropanol and analyzed. ImageJ software (National Institutes of Health, version no. $1.51 \mathrm{j} 8$ ) was used to quantify the results of the oil red $\mathrm{O}$ staining of frozen liver sections. TGs were extracted 

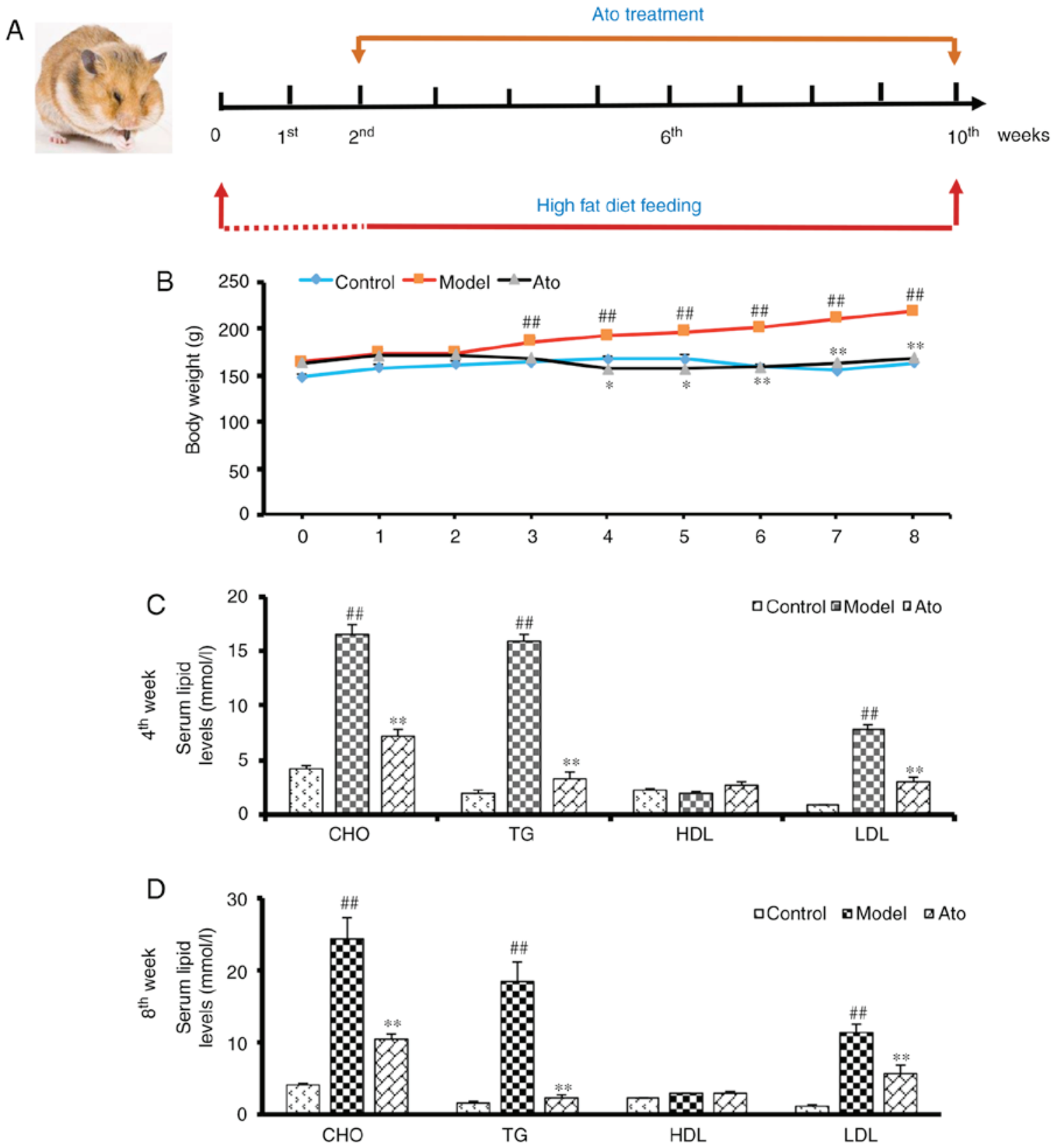

Figure 1. Effects of Ato on weight gain and serum lipid levels of golden hamsters fed a HFD. (A) Summary of the experimental design. (B) Body weight of golden hamsters in each group from 0 to 8 weeks of treatment. (C and D) CHO, TG, LDL-c and HDL-c in the serum of golden hamsters following Ato treatment for (C) 4 and (D) 8 weeks. Data are presented as the mean $\pm \mathrm{SEM}$ ( $\mathrm{n}=8 /$ group). ${ }^{\# \#} \mathrm{P}<0.01$ vs. control group; $\mathrm{P}<0.05,{ }^{* *} \mathrm{P}<0.01$ vs. model group. Ato, atorvastatin; HFD, high fat diet; CHO, cholesterol; TG, triglyceride; LDL, low density lipoprotein; HDL, high density lipoprotein.

from oil red O-stained HepG2 cells with 100\% isopropanol and were analyzed by measuring the optical density at $490 \mathrm{~nm}$.

Total RNA extraction and reverse transcription-quantitative $P C R(R T-q P C R)$ analysis. Total RNA was extracted from tissues using TRIzol. The purity and concentration of total RNA was measured using a spectrophotometer (Nanodrop 3000; Thermo Fisher Scientific, Inc.). Next, 1,000 ng total RNA was reverse transcribed using a PrimeScript RT Master $\operatorname{mix}\left(37^{\circ} \mathrm{C}\right.$ for $15 \mathrm{~min}, 85^{\circ} \mathrm{C}$ for $5 \mathrm{sec}$, then hold at $\left.4^{\circ} \mathrm{C}\right)$. The expression levels of adipose triglyceride lipase (ATGL), lipase E, hormone sensitive type (HSL), pyruvate dehydrogenase kinase 4 (PDK4), carnitine palmitoyltransferase 1A
(CPT1a), CPT1b, FA synthase (FASN), stearoyl-CoA desaturase (SCD1), diacylglycerol O-acyltransferase 1 (DGAT1), peroxisome proliferator activated receptor $\alpha$ (PPAR $\alpha)$, PPARG coactivator $1 \alpha$ (PPARGC1A) and GAPDH were analyzed using the CFX-96 Touch Thermocycler with a SYBR Green Master mix. The thermocycling conditions were as follows: Incubation at $95^{\circ} \mathrm{C}$ for $30 \mathrm{sec}$, followed by 40 cycles of $95^{\circ} \mathrm{C}$ for $5 \mathrm{sec}, 60^{\circ} \mathrm{C}$ for $30 \mathrm{sec}$ and $72^{\circ} \mathrm{C}$ for $30 \mathrm{sec}$, then dissociation stage. Primer sequences are presented in Table I. The results were normalized to GAPDH and calculated using the $2^{-\Delta \Delta C q}$ method (21).

Western blot analysis. Western blot assay was performed as previously described (22). Briefly, proteins were isolated from 
Table I. Sequences of the primers used for reverse transcription-quantitative PCR.

\begin{tabular}{lll}
\hline Gene name & \multicolumn{1}{c}{ Forward primer } & Reverse primer \\
\hline ATGL & CACTTTAGCTCCAAGGATGA & TGGTTCAGTAGGCCATTCCT \\
HSL & GGTGACACTCGCAGAAGACAATA & GCCGCCGTGCTGTCTCT \\
PDK4 & CACAGTTGAGCACCAAGA & AGCCTGACATAGAGTAGAGA \\
CPT1a & CCACTGATGAAGGAAGGAG & TAGTAGTTGCTGTTGACCAT \\
CPT1b & CTCCACAAGCCAGATTCC & ACTCACCGTCTCAGAACT \\
FASN & CTTGTCCAGGTTCGTGAG & TGTTGCTTCGGTGATGAG \\
SCD1 & CTTCACCACATTCTTCATCG & TCCCGTCTCCAGTTCTTT \\
DGAT1 & GGCATCATACTCCATCATCT & TCTCGGTAGTTCAGATTGTC \\
PPAR $\alpha$ & GAGAAAGCAAAACTGAAAGCAGAGA & GAAGGGCGGGTTATTGCTG \\
GAPDHC $1 \mathrm{~A}$ & TGGATGAAGACGGATTGC & GCGACTGTGGTTGTGTAT \\
\end{tabular}

ATGL, adipose triglyceride lipase; HSL, lipase E, hormone sensitive type; PDK4, pyruvate dehydrogenase kinase 4; CPT1a/b, carnitine palmitoyltransferase 1A/B; FASN, fatty acid synthase; SCD1, stearoyl-CoA desaturase; DGAT1, diacylglycerol O-acyltransferase 1; PPAR $\alpha$, peroxisome proliferator activated receptor $\alpha$; PPARGC1A, PPARG coactivator $1 \alpha$.

cells or tissue samples using RIPA buffer containing $50 \mathrm{mM}$ Tris- $\mathrm{HCl}$ (pH 7.4), 150 mM NaCl, 1\% NP-40, 0.5\% sodium deoxycholate and $0.1 \%$ SDS. The protein concentration was measured using BCA Protein Assay Reagent (Thermo Fisher Scientific, Inc.), and $50 \mu \mathrm{g}$ of protein samples were loaded into $10 \%$ gel and were separated by SDS-PAGE, and then transferred to a PVDF membrane. After blocking with 5\% non-fat milk in TBST for $2 \mathrm{~h}$ at room temperature, the blots were incubated with the primary antibodies at $4{ }^{\circ} \mathrm{C}$ overnight, including AMPK $\alpha$ (CST; 1:1,000; cat. no. 2532), phosphorylated (p-)AMPK $\alpha$ (CST; 1:1,000; cat. no. 2535), Akt (CST; 1:1,000; cat. no. 9272), p-Akt (CST; 1:1,000; cat. no. 4060), PPAR $\alpha$ (Abcam; 1:1,000; cat. no. ab61182), PGC1 $\alpha$ (Abcam; 1:1,000; cat. no. ab54481) and GAPDH (Abcam; 1:2,000; cat. no. ab8245). After washing with TBST, membranes were probed with secondary antibody labeled with horseradish peroxidase (Thermo Fisher Scientific, Inc.; 1;5,000; cat. no. 31460) for $1 \mathrm{~h}$ at room temperature. The protein bands were detected using enhanced chemiluminescent substrate (Thermo Fisher Scientific, Inc.; cat. no. 34580) and quantified using Image Lab 3.0 (Bio-Rad Laboratories, Inc.).

Statistical analysis. Data are presented as the mean \pm SEM. The statistical significance of differences among groups was assessed by one-way ANOVA followed by the Student-Newman-Keuls test. $\mathrm{P}<0.05$ was considered to indicate a statistically significant difference.

\section{Results}

Ato decreases the body weight and serum lipid levels in golden hamsters. During the 8 weeks of treatment with Ato (Fig. 1A), the body weights of the golden hamsters from each group were measured. Compared with the control animals, HFD-fed hamsters showed increased body weight, which was significantly attenuated by Ato treatment, indicating that Ato suppressed HFD-induced weight gain (Fig. 1B).
Serum lipid levels were analyzed at the end of the fourth and eighth weeks of treatment with Ato. After 4 weeks of treatment, the serum levels of CHO, TG and LDL in the model group were markedly increased by $285.33,680.50$ and $735.72 \%$, respectively, in comparison with the control group, indicating that the hamsters in the model group exhibited hyperlipidemia. Following 4 weeks of Ato treatment, CHO, TG and LDL levels were decreased by $55.68,79.16$ and $60.78 \%$, respectively compared with those in the model group (Fig. 1C). After 8 weeks of Ato treatment, in line with the results of the fourth week, Ato significantly reduced the increased serum levels of CHO, TG and LDL induced by the administration of HFD for 10 weeks (Fig. 1D).

Ato decreases the serum levels of insulin and CRP. Previous studies have shown that hyperinsulinemia and increased CRP levels are associated with NAFLD $(23,24)$. The golden hamsters fed with HFD for 6 weeks exhibited increased serum insulin and CRP levels (Fig. 2A). After 10 weeks of HFD, the serum levels of insulin and CRP in the model group were increased by 101.35 and $80.28 \%$ respectively, and these increases were significantly attenuated following Ato treatment (Fig. 2B).

Ato reduces the accumulation of lipids in the liver. After 10 weeks of HFD, macroscopic observation of the liver tissue indicated an increased accumulation of lipids in the model group compared with the control group (Fig. 3A), and this effect was alleviated following treatment with Ato. Additionally, chronic HFD increased liver weight (5.41 vs. 4.56/100 g body weight; Fig. 3B) and hepatic TG content (15.40 vs. $8.48 \mu \mathrm{mol} / \mathrm{g}$; Fig. 3C) compared with the control group. After 8 weeks of Ato treatment, the increased liver weight and TG content were reduced by 9.20 and $29.22 \%$, respectively, indicating that Ato effectively inhibited fat accumulation in the liver.

Possible pathological alterations were further investigated. Results from H\&E staining (Fig. 4A-C) showed that HFD increased hepatic fat accumulation, as the majority of hepatocytes in the livers of HFD-fed hamsters were increased 

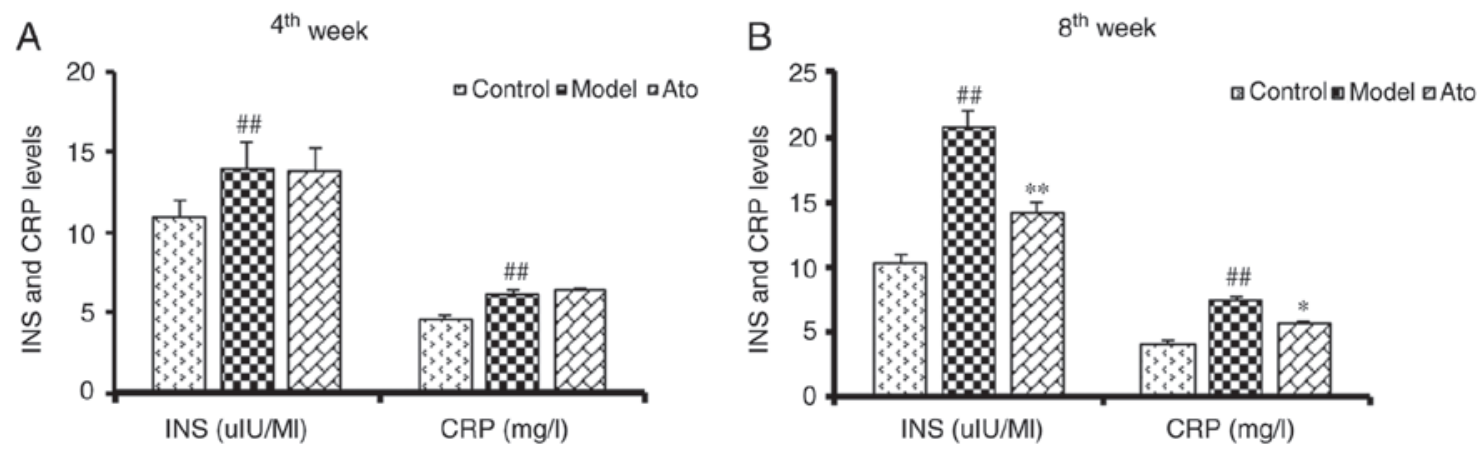

Figure 2. Effect of Ato on the serum levels of INS and CRP. (A) Serum INS and CRP levels of golden hamsters following Ato treatment for 4 weeks. (B) Serum INS and CRP levels of golden hamsters following Ato treatment for 8 weeks. Data are presented as the mean \pm SEM ( $\mathrm{n}=8 /$ group). ${ }^{\# P} \mathrm{P}<0.01 \mathrm{vs.}$. control group; ${ }^{*} \mathrm{P}<0.05,{ }^{* *} \mathrm{P}<0.01$ vs. model group. Ato, atorvastatin; INS, insulin; CRP, C-reactive protein.

A

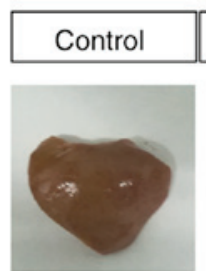

B

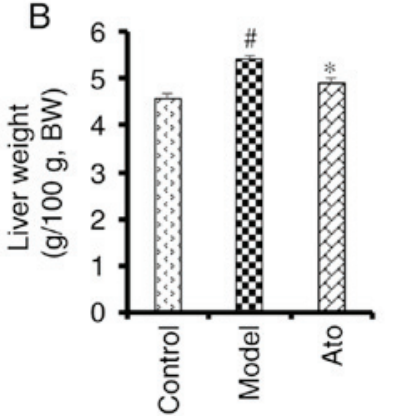

Model

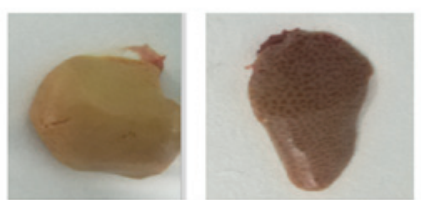

C

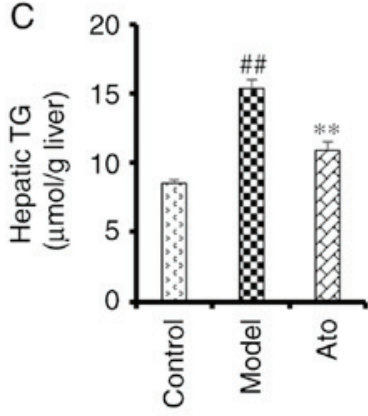

Figure 3. Effect of Ato on liver weight and hepatic TG in HFD-fed golden hamsters. (A) Representative images of livers from different groups. (B) Relative liver weight in each group. (C) Hepatic TG level in golden hamsters following Ato treatment for 8 weeks. Data are presented as the mean \pm SEM ( $=8 /$ group). ${ }^{~} \mathrm{P}<0.05,{ }^{\# \#} \mathrm{P}<0.01$ vs. control group; ${ }^{*} \mathrm{P}<0.05$, ${ }^{* *} \mathrm{P}<0.01$ vs. model group. Ato, atorvastatin; TG, triglyceride; HFD, high fat diet; BW, body weight.

in volume due to the accumulation of fat. Numerous single large adipocytes exhibited displaced nuclei, and ballooning degeneration was evident, and the hepatocytes, which exhibited cytoplasmic vacuolation, were swollen (Fig. 4B). Oil red $\mathrm{O}$ staining results showed that Ato treatment for 8 weeks reduced the accumulation of FAs in the liver compared with that in the model group (Fig. 4D-F; Fig. S1). The present results suggested that Ato exhibited the ability to inhibit the progression of NAFLD.

Ato alleviates NAFLD by promoting the expression of genes involved in lipolysis and inhibiting the expression of genes involved in fat synthesis. HFD significantly decreased the hepatic mRNA levels of ATGL and HSL in hamsters to 40 and $58 \%$, respectively, compared with the control group. In addition, HFD suppressed a subset of genes involved in $\beta$ oxidation, namely CPT1a, CPT1b and PDK4 (Fig. 5A). Ato reversed the HFD-induced decrease in the hepatic mRNA levels of ATGL and HSL by $225 \%(\mathrm{P}<0.01)$ and $122.41 \%$ $(\mathrm{P}=0.09)$, respectively, and upregulated the reduced mRNA levels of CPT1a, CPT1b and PDK4 induced by HFD treatment, thus promoting adipose lipolysis. Additionally, HFD increased the expression levels of FASN and DGAT1 compared with those in the control group, and these increases were attenuated by Ato treatment (Fig. 5B). The present results suggested that Ato could increase fat oxidation in the NAFLD model.

Ato inhibits lipid accumulation via the AMPK-dependent upregulation of PPAR $\alpha$ and PGCl $\alpha$. AMPK is a key molecule involved in the regulation of biological metabolism, and it has been reported to be associated with diabetes and other metabolic diseases. Accumulating evidence has shown that statins can activate the AMPK pathway $(25,26)$. Therefore, the present study aimed to determine whether Ato exerts its protective roles by regulating AMPK (Fig. 6). Western blotting results showed that the $\mathrm{p}$-AMPK/AMPK and p-Akt/Akt ratios in the livers of HFD-fed hamsters were decreased to 56 and $31 \%$, respectively, of those in the control group (Fig. 6A). Ato treatment for 8 weeks significantly upregulated the p-AMPK/AMPK and p-Akt/Akt ratios in the liver by 1.91- and 2.42-fold, respectively, compared with those in the model group (Fig. 6B). In addition, Ato was found to suppress the lipid accumulation in HepG2 cells induced by treatment with $200 \mu \mathrm{M}$ PA for $24 \mathrm{~h}$ (Fig. 6D). In line with the present in vivo results, the phosphorylation ratio of AMPK was decreased in PA-treated HepG2 cells, but was upregulated following treatment with $20 \mu \mathrm{M}$ Ato for $24 \mathrm{~h}$ (Fig. $6 \mathrm{E}$ and F). Treatment with $10 \mu \mathrm{M}$ Compound $\mathrm{C}$, an AMPK inhibitor, for $24 \mathrm{~h}$ significantly suppressed the protective effects of Ato as an inhibitor of lipid accumulation in PA-treated HepG2 cells. Collectively, the present results indicate that AMPK is one of the main targets of Ato involved in the preventive effects of Ato on NAFLD.

Numerous previous studies have indicated that PPAR $\alpha$ is downstream of AMPK and Akt, and that its expression level is regulated by Akt (27-29). Therefore, the mRNA and protein expression levels of PPAR $\alpha$ and PPARGC1A were investigated in the liver tissues of the hamsters in the present study. Western blotting results showed that the protein expression levels of PPAR $\alpha$ and PGCl $\alpha$ were decreased to 59.75 and $72.26 \%$, respectively, compared with those in the control 

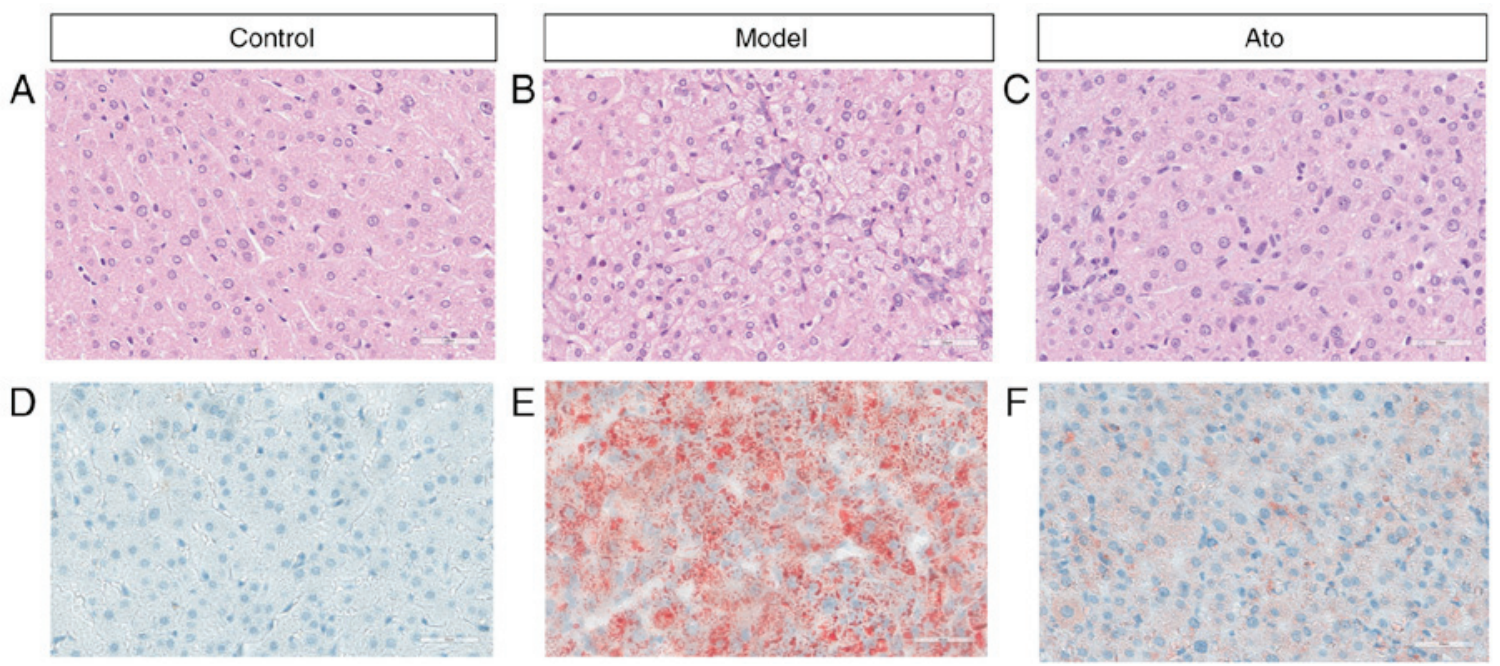

Figure 4. Effect of Ato on lipid accumulation in livers of HFD-fed golden hamsters. (A-C) Hematoxylin and eosin staining of liver tissues from each group under a light microscope (magnification, x200). (D-F) Oil red O staining of liver tissues from each group under a light microscope (magnification, x200). Ato, atorvastatin; HFD, high fat diet.

A
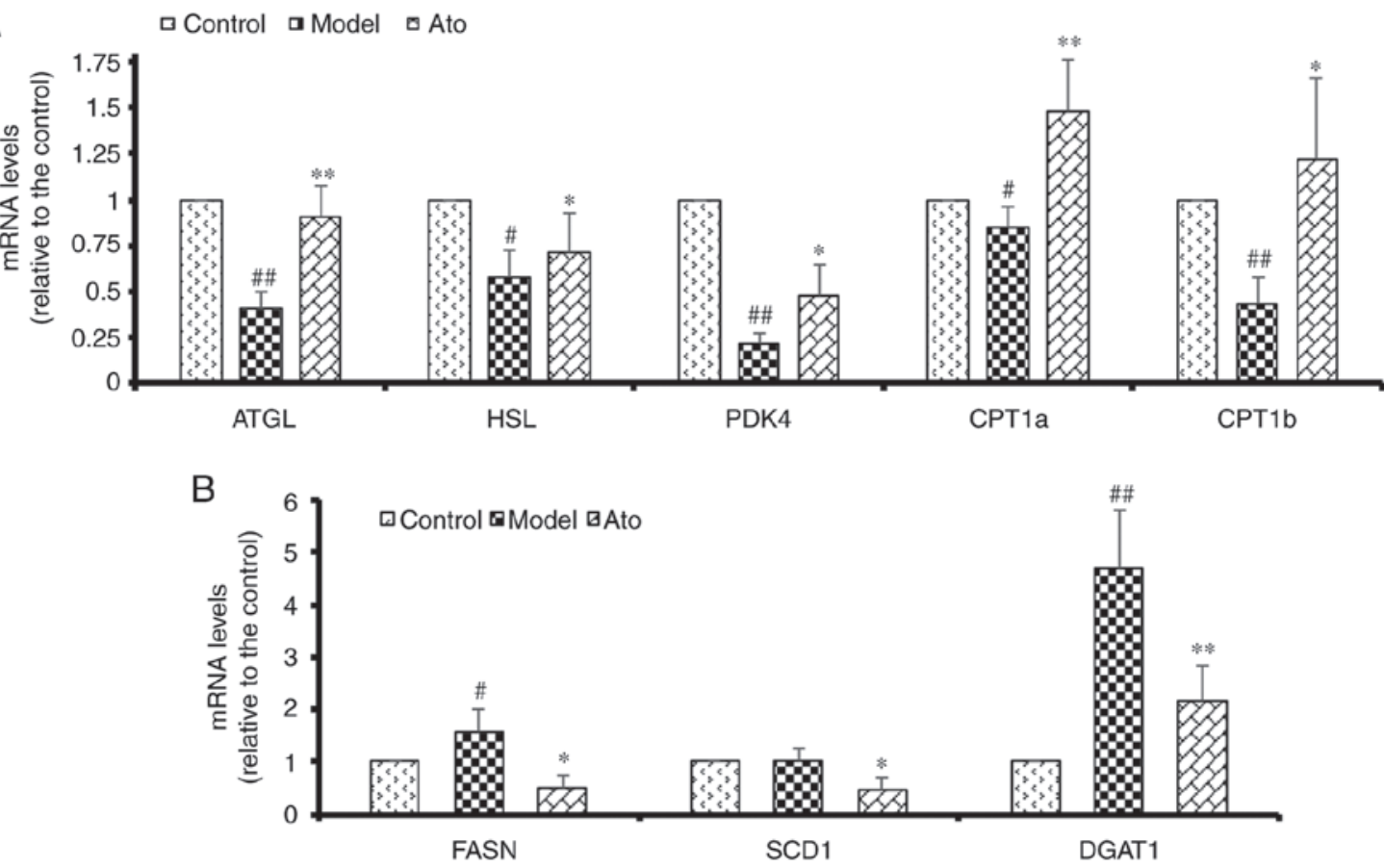

Figure 5. Effect of Ato on the mRNA levels of genes involved in lipid metabolism. (A) mRNA levels of genes associated with lipolysis and $\beta$-oxidation. (B) mRNA levels of genes associated with fatty acid synthesis. Data are presented as the mean $\pm \mathrm{SEM}$ ( $\mathrm{n}=8 / \mathrm{group}$ ). ${ }^{\#} \mathrm{P}<0.05$, ${ }^{\#} \mathrm{P}<0.01 \mathrm{vs}$. control group; ${ }^{*} \mathrm{P}<0.05,{ }^{* *} \mathrm{P}<0.01$ vs. model group. Ato, atorvastatin; ATGL, adipose triglyceride lipase; HSL, lipase E, hormone sensitive type; PDK4, pyruvate dehydrogenase kinase 4; CPT1a/CPT1b, carnitine palmitoyltransferase 1A/B; FASN, fatty acid synthase; SCD1, stearoyl-CoA desaturase; DGAT1, diacylglycerol O-acyltransferase 1.

group $(\mathrm{P}<0.05)$. Treatment with Ato for 8 weeks upregulated the protein expression levels of PPAR $\alpha$ and PGC1 $\alpha$ by 1.66- and 2.20-fold, respectively, compared with those in the model group $(\mathrm{P}<0.05$; Fig. $6 \mathrm{C})$. Consistent with the western blotting results, the RT-qPCR results showed that Ato significantly promoted the mRNA expression levels of PPAR $\alpha$ and PPARGC1A ( $\mathrm{P}<0.05$; Fig. 6G). Collectively, the present results indicate that Ato exerted its protective effects in preventing NAFLD via the AMPK-dependent upregulation of PPAR $\alpha$ and PGC1 $\alpha$.

\section{Discussion}

Statins are the most commonly prescribed drugs for the attenuation of hypercholesterolemia by blocking HMG-CoA reductase (30). Recent clinical studies have shown that statins can reduce hepatic lipid accumulation, thereby alleviating NAFLD (31), but the mechanisms underlying this effect are poorly understood. Animal models of NAFLD induced by $\operatorname{HFD}(32,33)$ have been widely used to examine the pathogenesis of NAFLD and to investigate new treatment 
A
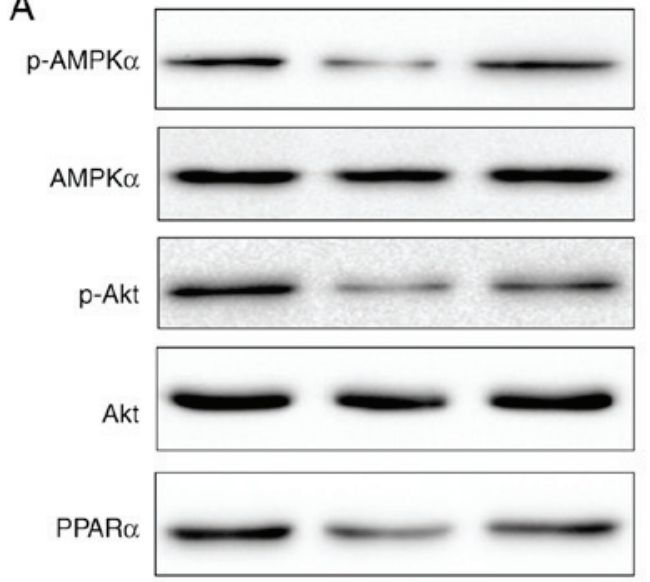

PGC1 $\longrightarrow-\ldots$

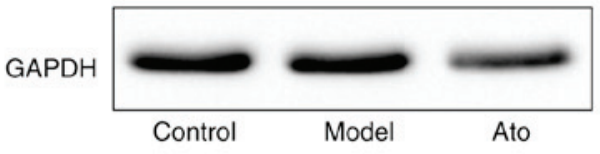

E

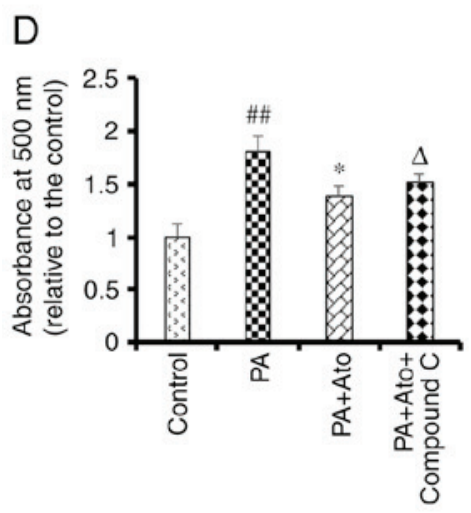

$\mathrm{G}$

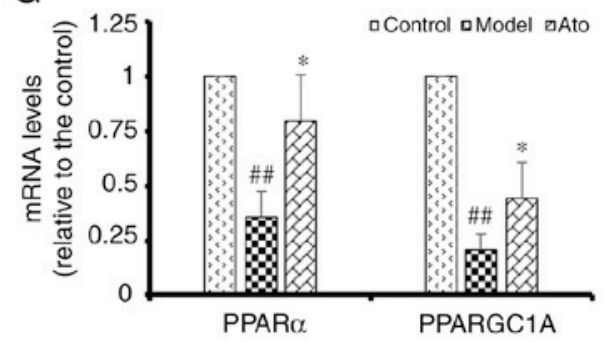

B

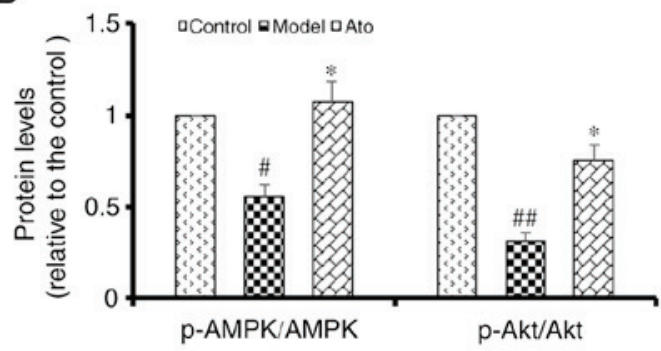

C

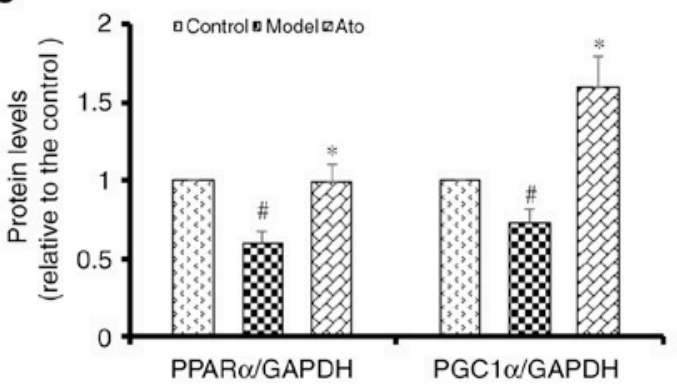

$\mathrm{F}$

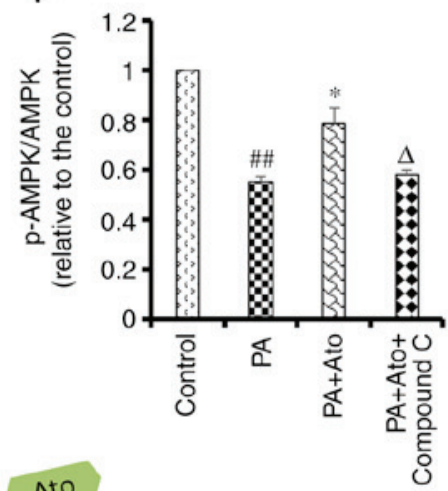

$\mathrm{H}$

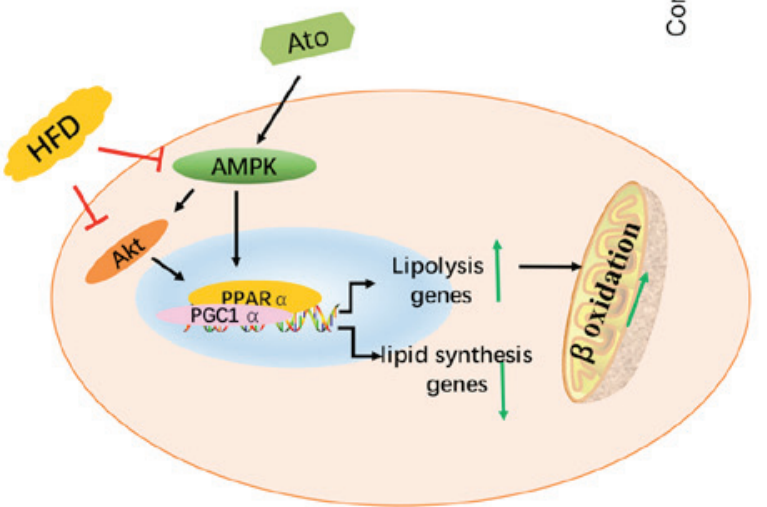

Figure 6. Ato inhibits lipid accumulation by promoting the AMPK signaling pathway. (A) Representative images of p-AMPK, AMPK, p-Akt, Akt, PPAR $\alpha$ and PGCl $\alpha$ western blots. (B) Relative p-AMPK/AMPK, p-Akt/Akt and (C) PPAR $\alpha /$ GAPDH and PGCl $\alpha /$ GAPDH protein levels are presented. ${ }^{\# P}<0.05$, ${ }^{\# \#} \mathrm{P}<0.01$ vs. control group; ${ }^{*} \mathrm{P}<0.05$ vs. model group. (D) Lipid accumulation in HepG2 cells, quantified by the absorbance value of oil red O reagent at $490 \mathrm{~nm}$. Data are presented as the mean \pm SEM $(n=6)$. (E) Representative images of p-AMPK and AMPK in PA-treated HepG2 cells with or without Ato and Compound C treatment. (F) Relative p-AMPK/AMPK protein levels. Data are presented as the mean $\pm \mathrm{SEM}(\mathrm{n}=3){ }^{\#}{ }^{\#} \mathrm{P}<0.01$ vs. control group; ${ }^{*} \mathrm{P}<0.05$ vs. $\mathrm{PA}$ group; ${ }^{\circ} \mathrm{P}<0.05$ vs. PA + Ato group. (G) mRNA levels of PPAR $\alpha$ and PPARGC1A in each group. Data are presented as the mean \pm SEM (n=3/group). ${ }^{\# \#} \mathrm{P}<0.01$ vs. control group; "P<0.05 vs. model group. (H) Schematic illustration showing the suggested mechanisms underlying the protective effect of Ato against non-alcoholic fatty liver disease. Ato, atorvastatin; p-, phosphorylated; PPAR $\alpha$, peroxisome proliferator activated receptor $\alpha$; AMPK, AMP-activated protein kinase; PGC1 $\alpha$, peroxisome proliferator-activated receptor- $\gamma$ coactivator $1 \alpha$; PPARGC1A, PPARG coactivator $1 \alpha$; PA, palmitate.

strategies (34). HFD-induced liver disease, in contrast with rifampicin-isoniazid-induced hepatotoxicity (35), not only leads to chronic inflammation and liver fibrosis, but also disrupts endoplasmic reticulum calcium homeostasis (36-38).
In the present study, hamsters were given a HFD for 10 weeks and developed hepatic steatosis with high levels of TG, TCH, insulin and CRP, phenocopying the clinical features of human NAFLD. Following Ato treatment, the increased body weight 
and elevated serum levels of TCH, TG, LDL, insulin and CRP identified in HFD-fed hamsters were decreased. Additionally, the relative liver weight of HFD-fed hamsters treated with Ato was markedly decreased compared with that of the model group. Morphologically, the livers of hamsters in the model group exhibited numerous large lipid droplets and various defects compared with the control group. However, the livers of HFD-fed hamsters treated with Ato exhibited fewer lipid droplets and an improved liver morphology, indicating that Ato exhibited beneficial effects in suppressing lipid accumulation and attenuating the disruption of liver structure.

The prevalence of NAFLD in China is increasing. In 2014, a meta-analysis of epidemiologic studies revealed that the overall prevalence of NAFLD was $\sim 20 \%$ in China (39). NAFLD has been identified to be associated with obesity, dyslipidemia, insulin resistance and T2DM (40). Due to the increase in metabolic risk factors, patients suffering from NAFLD are at higher risk of developing cardiovascular diseases, which are associated with increased morbidity and mortality (41). NAFLD contributes to the development of T2DM, non-alcoholic steatohepatitis and hepatocellular carcinoma (42). However, the number of targeted drugs to treat NAFLD remain limited. In clinical settings, statins are widely used to reduce the serum levels of lipids, which is considered to suppress NAFLD. However, the number of studies investigating the protective effects of Ato on NAFLD remain limited and its underlying molecular mechanisms are unclear. Therefore, to investigate these questions, an animal model of NAFLD was constructed in the present study, in which golden hamsters were fed a HFD to induce NAFLD. Ato treatment not only decreased the serum levels of TG, TCH, LDL, CRP and insulin, but also attenuated lipid accumulation and pathological alterations, indicating that Ato exhibited the potential to treat NAFLD.

The pathogenesis of NAFLD is complex and involves numerous signaling pathways, including the PI3K/Akt, Toll-like receptor (TLR), apoptosis and mTOR pathways $(43,44)$. Deficient PI3K activity leads to an increase in intracellular FA-derived metabolites (45). TLR-4 can activate X-box binding protein-1 and thereby promote the progression of NAFLD (46). Increases in active caspase 2, active caspase 3 and apoptosis have been observed in the livers of patients with NAFLD (47). Metabolic abnormality of lipids in the liver may cause insulin resistance, inflammation and apoptosis (48-50).

AMPK regulates numerous metabolic pathways and may have therapeutic potential in the treatment of obesity, insulin resistance, T2DM and NAFLD. Activation of AMPK can inhibit acetyl CoA carboxylase (ACC), which plays crucial roles in lipogenesis. AMPK also regulates FA metabolism in the liver via the regulation of total mitochondrial content and function. Recent studies have indicated that statins may be able to alleviate numerous diseases by regulating the AMPK-mediated pathway, including neurotoxicity, myocardial fibrosis and endoplasmic reticulum stress (51-53). These previous studies suggest that Ato may protect against NAFLD by increasing the activity of the AMPK signaling pathway.

As an energy sensor that maintains cellular energy homeostasis, AMPK has been reported to positively regulate FA oxidation by activating PPAR $\alpha$ and PGC1 $\alpha$ (54). Additionally, there is evidence to indicate that AMPK activates the PI3K/Akt pathway by inhibiting the phosphorylation of insulin receptor substrate-1 (Ser $\left.{ }^{636 / 639}\right)(55-57)$. Hinoi et al (58) found that the PI3K/Akt pathway could indirectly promote the nuclear translocation of $\mathrm{PGCl} \alpha$, which serves crucial roles in FA oxidation. Consistent with a previous study (59), the livers of HFD-fed hamsters in the present study exhibited a significant reduction in AMPK phosphorylation levels compared with the control group. Akt phosphorylation, as well as PPAR $\alpha$ and PGC1 $\alpha$ protein expression levels were also reduced in the model group compared with the control. However, Ato treatment for 8 weeks significantly reversed the decreased levels of p-AMPK, p-Akt, PPAR $\alpha$ and PGC1 $\alpha$, indicating that Ato is able to regulate the expression of AMPK and its downstream targets.

Activation of FA $\beta$-oxidation facilitates the metabolism of lipids in the liver. PPAR $\alpha$ has been indicated to stimulate FA $\beta$-oxidation by increasing the expression level of PDK4 (60). Additionally, PPAR $\alpha$ controls the constitutive expression of mitochondrial FA $\beta$-oxidation enzymes (61) and increases the level of uncoupling protein 2 in mitochondria (62). Since Ato upregulated PPAR $\alpha$ via AMPK and its downstream targets, the present study also investigated the mRNA levels of several genes involved in lipid metabolism, including lipogenesis and $\beta$-oxidation. The results revealed that the HFD-induced inhibition of ATGL, HSL, PDK4, CPT1a and CPT1b expression levels could be effectively attenuated by Ato treatment. Collectively, the present data indicate that Ato may promote lipid metabolism via the AMPK-dependent upregulation of PPAR $\alpha$, PGCl $\alpha$ and their target genes.

However, the present study presents certain limitations. The impact the Ato on control mice was not explored in this study. Although Ato may protect against NAFLD by activating AMPK and its target genes, it may have multiple targets in addition to AMPK and HMG-CoA reductase. Furthermore, in vitro experiments are required to investigate whether the protective effects of Ato are neutralized following the knockdown of AMPK using small interfering RNA or adenoviral means. Future studies conducted by the present team will aim to investigate this aspect.

In conclusion, the results of the present study suggest that Ato could effectively prevent the progression of NAFLD in a hamster model, as evidenced by its ability to attenuate the HFD-induced increases in serum levels of lipids, insulin and $\mathrm{CRP}$, and by the reduction of lipid accumulation in the liver. Mechanistically, the results also indicate that Ato inhibited fat accumulation in the liver via the AMPK-dependent upregulation of PPAR $\alpha, \mathrm{PGCl} \alpha$ and their target genes, which are associated with $\beta$-oxidation. In conclusion, the present study suggests the potential of Ato as a therapeutic drug for the clinical treatment of NAFLD.

\section{Acknowledgements}

Not applicable.

\section{Funding}

This study was supported by The National Science Foundation for Young Scientists of China (grant no. 81803803). 


\section{Availability of data and materials}

The datasets used and/or analyzed during the current study are available from the corresponding author on reasonable request.

\section{Authors' contributions}

BZ, GS and XS designed the study. BZ, CZ, XZ, NL and $\mathrm{ZD}$ performed the experiments. $\mathrm{CZ}$ analyzed the data and BZ wrote the manuscript. All authors discussed, edited and approved the final version of the manuscript.

\section{Ethics approval and consent to participate}

The animal protocol was approved by The Experimental Laboratory Animal Committee of Chinese Academy of Medical Sciences and Peking Union Medical College.

\section{Patient consent for publication}

Not applicable.

\section{Competing interests}

The authors declare that they have no competing interests.

\section{References}

1. Younossi ZM, Koenig AB, Abdelatif D, Fazel Y, Henry L and Wymer M: Global epidemiology of nonalcoholic fatty liver disease-Meta-analytic assessment of prevalence, incidence, and outcomes. Hepatology 64: 73-84, 2016.

2. Anderson EL, Howe LD, Jones HE, Higgins JP, Lawlor DA and Fraser A: The prevalence of non-alcoholic fatty liver disease in children and adolescents: A systematic review and meta-analysis PLoS One 10: e0140908, 2015.

3. Alisi A, Feldstein AE, Villani A, Raponi M and Nobili V: Pediatric nonalcoholic fatty liver disease: A multidisciplinary approach. Nat Rev Gastroenterol Hepatol 9: 152-161, 2012.

4. Ekstedt M, Hagström H, Nasr P, Fredrikson M, Stål P, Kechagias S and Hultcrantz R: Fibrosis stage is the strongest predictor for disease-specific mortality in NAFLD after up to 33 years of follow-up. Hepatology 61: 1547-1554, 2015.

5. Angulo P, Kleiner DE, Dam-Larsen S, Adams LA, Bjornsson ES, Charatcharoenwitthaya P, Mills PR, Keach JC, Lafferty HD, Stahler A, et al: Liver fibrosis, but no other histologic features, is associated with long-term outcomes of patients with nonalcoholic fatty liver disease. Gastroenterology 149: 389-397.e310, 2015.

6. Tanaka S, Hikita H, Tatsumi T, Sakamori R, Nozaki Y, Sakane S, Shiode Y, Nakabori T, Saito Y, Hiramatsu N, et al: Rubicon inhibits autophagy and accelerates hepatocyte apoptosis and lipid accumulation in nonalcoholic fatty liver disease in mice. Hepatology 64: 1994-2014, 2016.

7. Aller R, Fernández-Rodríguez C, Lo Iacono O, Bañares R, Abad J, Carrión JA, García-Monzón C, Caballería J, Berenguer M, Rodríguez-Perálvarez M, et al: Consensus document. Management of non-alcoholic fatty liver disease (NAFLD). Clinical practice guideline. Gastroenterol Hepatol 41: 328-349, 2018 (In English, Spanish).

8. Marchesini G, Brizi M, Bianchi G, Tomassetti S, Bugianesi E, Lenzi M, McCullough AJ, Natale S, Forlani G and Melchionda N: Nonalcoholic fatty liver disease: A feature of the metabolic syndrome. Diabetes 50: 1844-1850, 2001.

9. Yamasaki T and Tomita K: Relationship between hyperuricemia and metabolic syndrome. Nihon Rinsho 66: 766-770, 2008 (In Japanese).

10. Kim JY, Garcia-Carbonell R, Yamachika S, Zhao P, Dhar D, Loomba R, Kaufman RJ, Saltiel AR and Karin M: ER stress drives lipogenesis and steatohepatitis via caspase-2 activation of S1P. Cell 175: 133-145.e15, 2018
11. Park JG, Xu X, Cho S, Hur KY, Lee MS, Kersten S and Lee AH: CREBH-FGF21 axis improves hepatic steatosis by suppressing adipose tissue lipolysis. Sci Rep 6: 27938, 2016.

12. Stavropoulos K, Imprialos K, Pittaras A, Faselis C, Narayan P and Kokkinos P: Lifestyle modifications in non-alcoholic fatty liver disease and non-alcoholic steatohepatitis. Curr Vasc Pharmacol 16: 239-245, 2018.

13. Mathews SE, Kumar RB and Shukla AP: Nonalcoholic steatohepatitis, obesity, and cardiac dysfunction. Curr Opin Endocrinol Diabetes Obes 25: 315-320, 2018.

14. Ye YC, Zhao XL and Zhang SY: Use of atorvastatin in lipid disorders and cardiovascular disease in Chinese patients. Chin Med J (Engl) 128: 259-266, 2015.

15. Bakker-Arkema RG, Davidson MH, Goldstein RJ, Davignon J, Isaacsohn JL, Weiss SR, Keilson LM, Brown WV, Miller VT, Shurzinske LJ and Black DM: Efficacy and safety of a new HMG-CoA reductase inhibitor, atorvastatin, in patients with hypertriglyceridemia. JAMA 275: 128-133, 1996.

16. Amarenco P, Bogousslavsky J, Callahan A III, Goldstein LB, Hennerici M, Rudolph AE, Sillesen H, Simunovic L, Szarek M, Welch KM, et al: High-dose atorvastatin after stroke or transient ischemic attack. N Engl J Med 355: 549-559, 2006.

17. Taniguti EH, Ferreira YS, Stupp IJV, Fraga-Junior EB, Doneda DL, Lopes L, Rios-Santos F, Lima E, Buss ZS, Viola GG and Vandresen-Filho S: Atorvastatin prevents lipopolysaccharide-induced depressive-like behaviour in mice. Brain Res Bull 146: 279-286, 2019

18. Cioboată R, Găman A, Traşcă D, Ungureanu A, Docea AO, Tomescu P, Gherghina F, Arsene AL, Badiu C, Tsatsakis AM, et al: Pharmacological management of non-alcoholic fatty liver disease: Atorvastatin versus pentoxifylline. Exp Ther Med 13: 2375-2381, 2017.

19. Brenachot X, Ramadori G, Ioris RM, Veyrat-Durebex C, Altirriba J, Aras E, Ljubicic S, Kohno D, Fabbiano S, Clement S, et al: Hepatic protein tyrosine phosphatase receptor gamma links obesity-induced inflammation to insulin resistance. Nat Commun 8: 1820, 2017.

20. Lerat H, Honda M, Beard MR, Loesch K, Sun J, Yang Y, Okuda M, Gosert R, Xiao SY, Weinman SA and Lemon SM: Steatosis and liver cancer in transgenic mice expressing the structural and nonstructural proteins of hepatitis $\mathrm{C}$ virus. Gastroenterology 122: 352-365, 2002.

21. Livak KJ and Schmittgen TD: Analysis of relative gene expression data using real-time quantitative PCR and the 2(-Delta Delta C(T)) method. Methods 25: 402-408, 2001.

22. Li M, Tang Y, Wu L, Mo F, Wang X, Li H, Qi R, Zhang H, Srivastava A and Ling C: The hepatocyte-specific HNF4alpha/miR-122 pathway contributes to iron overload-mediated hepatic inflammation. Blood 130: 1041-1051, 2017.

23. Nuñez-Durán E, Aghajan M, Amrutkar M, Sütt S, Cansby E, Booten SL, Watt A, Ståhlman M, Stefan N, Häring HU, et al: Serine/threonine protein kinase 25 antisense oligonucleotide treatment reverses glucose intolerance, insulin resistance, and nonalcoholic fatty liver disease in mice. Hepatol Commun 2: 69-83, 2018.

24. Lee J, Yoon K, Ryu S, Chang Y and Kim HR: High-normal levels of hs-CRP predict the development of non-alcoholic fatty liver in healthy men. PLoS One 12: e0172666, 2017.

25. Wang JC, Li XX, Sun X, Li GY, Sun JL, Ye YP, Cong LL, Li WM, Lu SY, Feng J, Liu PJ, et al: Activation of AMPK by simvastatin inhibited breast tumor angiogenesis via impeding HIF-1alpha-induced pro-angiogenic factor. Cancer Sci 109: 1627-1637, 2018

26. Han JS, Sung JH and Lee SK: Inhibition of cholesterol synthesis in HepG2 cells by GINST-decreasing HMG-CoA reductase expression via AMP-activated protein kinase. J Food Sci 82: 2700-2705, 2017.

27. Dihingia A, Bordoloi J, Dutta P, Kalita J and Manna P: Hexane-Isopropanolic Extract of Tungrymbai, a North-East Indian fermented soybean food prevents hepatic steatosis via regulating AMPK-mediated SREBP/FAS/ACC/HMGCR and PPARalpha/CPT1A/UCP2 pathways. Sci Rep 8: 10021, 2018.

28. Gorowska-Wojtowicz E, Dutka P, Kudrycka M, Pawlicki P, Milon A, Plachno BJ, Tworzydlo W, Pardyak L, Kaminska A, Hejmej A, et al: Regulation of steroidogenic function of mouse Leydig cells: G-coupled membrane estrogen receptor and peroxisome proliferator-activated receptor partnership. J Physiol Pharmacol 69: 2018 
29. Muthukumaran P, Thiyagarajan G, Arun Babu R and Lakshmi BS: Raffinose from Costus speciosus attenuates lipid synthesis through modulation of PPARs/SREBP1c and improves insulin sensitivity through PI3K/AKT. Chem Biol Interact 284: 80-89, 2018.

30. Sirtori CR: The pharmacology of statins. Pharmacol Res 88 : 3-11, 2014

31. Athyros VG, Boutari C, Stavropoulos K, Anagnostis P, Imprialos KP, Doumas M and Karagiannis A: Statins: An under-appreciated asset for the prevention and the treatment of NAFLD or NASH and the related cardiovascular risk. Curr Vase Pharmacol 16: 246-253, 2018.

32. Xue L, Lu X, He J, Zhang T, Wu X, Zhang Y, Wang N, An Z, $\mathrm{Xu} \mathrm{J}$ and Geng Y: Serum CK 18-M30 reflect liver pathological severity during NAFLD progression in a rat model. Pathol Res Pract 214: 1778-1786, 2018.

33. Zhou D, Pan Q, Xin FZ, Zhang RN, He CX, Chen GY, Liu C, Chen YW and Fan JG: Sodium butyrate attenuates high-fat diet-induced steatohepatitis in mice by improving gut microbiota and gastrointestinal barrier. World J Gastroenterol 23: 60-75, 2017.

34. Ou TH, Tung YT, Yang TH and Chien YW: Melatonin improves fatty liver syndrome by inhibiting the lipogenesis pathway in hamsters with high-fat diet-induced hyperlipidemia. Nutrients 11 : E748, 2019.

35. Nwidu LL and Teme RE: Hot aqueous leaf extract of Lasianthera africana (Icacinaceae) attenuates rifampicin-isoniazid-induced hepatotoxicity. J Integr Med 16: 263-272, 2018.

36. Wires ES, Trychta KA, Bäck S, Sulima A, Rice KC and Harvey BK: High fat diet disrupts endoplasmic reticulum calcium homeostasis in the rat liver. J Hepatol 67: 1009-1017, 2017.

37. Chen Y, Qian Q and Yu J: Carbenoxolone ameliorates insulin sensitivity in obese mice induced by high fat diet via regulating the IkappaB- $\alpha / \mathrm{NF}-\kappa \mathrm{B}$ pathway and NLRP3 inflammasome. Biomed Pharmacother 115: 108868, 2019.

38. Goto R, Kamimura K, Shinagawa-Kobayashi Y, Sakai N, Nagoya T, Niwa Y, Ko M, Ogawa K, Inoue R, Yokoo T, et al: Inhibition of sodium glucose cotransporter 2 (SGLT2) delays liver fibrosis in a medaka model of nonalcoholic steatohepatitis (NASH). FEBS Open Bio 9: 643-652, 2019.

39. Li Z, Xue J, Chen P, Chen L, Yan S and Liu L: Prevalence of nonalcoholic fatty liver disease in mainland of China: A meta-analysis of published studies. J Gastroenterol Hepatol 29: 42-51, 2014

40. Caballería L, Pera G, Auladell MA, Torán P, Muñoz L, Miranda D, Alumà A, Casas JD, Sánchez C, Gil D, et al: Prevalence and factors associated with the presence of nonalcoholic fatty liver disease in an adult population in Spain. Eur J Gastroenterol Hepatol 22: 24-32, 2010.

41. Söderberg C, Stål P, Askling J, Glaumann H, Lindberg G, Marmur J and Hultcrantz R: Decreased survival of subjects with elevated liver function tests during a 28-year follow-up. Hepatology 51: 595-602, 2010

42. Wong CR, Nguyen MH and Lim JK: Hepatocellular carcinoma in patients with non-alcoholic fatty liver disease. World J Gastroenterol 22: 8294-8303, 2016.

43. Wang X: Down-regulation of lncRNA-NEAT1 alleviated the non-alcoholic fatty liver disease via mTOR/S6K1 signaling pathway. J Cell Biochem 119: 1567-1574, 2018.

44. Liu H, Li J, Tillman B, Morgan TR, French BA and French SW: TLR3/4 signaling is mediated via the NFkappaB-CXCR4/7 pathway in human alcoholic hepatitis and non-alcoholic steatohepatitis which formed Mallory-Denk bodies. Exp Mol Pathol 97: 234-240, 2014.

45. Kim JK, Fillmore JJ, Chen Y, Yu C, Moore IK, Pypaert M, Lutz EP, Kako Y, Velez-Carrasco W, Goldberg IJ, et al: Tissue-specific overexpression of lipoprotein lipase causes tissue-specific insulin resistance. Proc Natl Acad Sci USA 98 7522-7527, 2001.

46. Ye D, Li FY, Lam KS, Li H, Jia W, Wang Y, Man K, Lo CM, $\mathrm{Li} \mathrm{X}$ and $\mathrm{Xu}$ A: Toll-like receptor-4 mediates obesity-induced non-alcoholic steatohepatitis through activation of X-box binding protein-1 in mice. Gut 61: 1058-1067, 2012.

47. Ferreira DM, Castro RE, Machado MV, Evangelista T, Silvestre A, Costa A, Coutinho J, Carepa F, Cortez-Pinto H and Rodrigues CM: Apoptosis and insulin resistance in liver and peripheral tissues of morbidly obese patients is associated with different stages of non-alcoholic fatty liver disease. Diabetologia 54: 1788-1798, 2011
48. Smith GI, Shankaran M, Yoshino M, Schweitzer GG, Chondronikola M, Beals JW, Okunade AL, Patterson BW, Nyangau E, Field T, et al: Insulin resistance drives hepatic de novo lipogenesis in nonalcoholic fatty liver disease. J Clin Invest: 134165, Dec 5, 2019 (Epub ahead of print).

49. Wang Q, Ou Y, Hu G, Wen C, Yue S, Chen C, Xu L, Xie J, Dai $\mathrm{H}$, Xiao $\mathrm{H}$, et al: Naringenin attenuates nonalocholic fatty liver disease by downregulating NLRP3/NF-kappaB pathway in mice. Br J Pharmacol: Nov 23, 2019, (Epub ahead of print)

50. Kanda T, Matsuoka S, Yamazaki M, Shibata T, Nirei K, Takahashi H, Kaneko T, Fujisawa M, Higuchi T, Nakamura $\mathrm{H}$, et al: Apoptosis and non-alcoholic fatty liver diseases. World J Gastroenterol 24: 2661-2672, 2018.

51. Li HH, Lin CL and Huang CN: Neuroprotective effects of statins against amyloid $\beta$-induced neurotoxicity. Neural Regen Res 13: 198-206, 2018

52. Hermida N, Markl A, Hamelet J, Van Assche T, Vanderper A, Herijgers P, van Bilsen M, Hilfiker-Kleiner D, Noppe G, Beauloye C, et al: HMGCoA reductase inhibition reverses myocardial fibrosis and diastolic dysfunction through AMP-activated protein kinase activation in a mouse model of metabolic syndrome. Cardiovasc Res 99: 44-54, 2013.

53. Jia F, Wu C, Chen $\mathrm{Z}$ and Lu G: Atorvastatin inhibits homocysteine-induced endoplasmic reticulum stress through activation of AMP-activated protein kinase. Cardiovasc Ther 30: 317-325, 2012.

54. Lee WJ, Kim M, Park HS, Kim HS, Jeon MJ, Oh KS, Koh EH, Won JC, Kim MS, Oh GT, et al: AMPK activation increases fatty acid oxidation in skeletal muscle by activating PPARalpha and PGC-1. Biochem Biophys Res Commun 340: 291-295, 2006.

55. Tzatsos A and Kandror KV: Nutrients suppress phosphatidylinositol 3-kinase/Akt signaling via raptor-dependent mTOR-mediated insulin receptor substrate 1 phosphorylation. Mol Cell Biol 26: 63-76, 2006.

56. Chopra I, Li HF, Wang H and Webster KA: Phosphorylation of the insulin receptor by AMP-activated protein kinase (AMPK) promotes ligand-independent activation of the insulin signalling pathway in rodent muscle. Diabetologia 55: 783-794, 2012.

57. Zheng T, Yang X, Wu D, Xing S, Bian F, Li W, Chi J, Bai X, Wu G, Chen X, et al: Salidroside ameliorates insulin resistance through activation of a mitochondria-associated AMPK/PI3K/Akt/GSK3beta pathway. Br J Pharmacol 172: 3284-3301, 2015

58. Hinoi E, Iezaki T, Fujita H, Watanabe T, Odaka Y, Ozaki K and Yoneda Y: PI3K/Akt is involved in brown adipogenesis mediated by growth differentiation factor-5 in association with activation of the Smad pathway. Biochem Biophys Res Commun 450: 255-260, 2014

59. Docrat TF, Nagiah S, Krishnan A, Naidoo DB and Chuturgoon AA: Atorvastatin induces MicroRNA-145 expression in HEPG2 cells via regulation of the PI3K/AKT signalling pathway. Chem Biol Interact 287: 32-40, 2018.

60. Ferrari A, Longo R, Fiorino E, Silva R, Mitro N, Cermenati G, Gilardi F, Desvergne B, Andolfo A, Magagnotti C, et al: HDAC3 is a molecular brake of the metabolic switch supporting white adipose tissue browning. Nat Commun 8: 93, 2017.

61. Aoyama T, Peters JM, Iritani N, Nakajima T, Furihata K, Hashimoto T and Gonzalez FJ: Altered constitutive expression of fatty acid-metabolizing enzymes in mice lacking the peroxisome proliferator-activated receptor alpha (PPARalpha). J Biol Chem 273: 5678-5684, 1998.

62. Patterson AD, Shah YM, Matsubara T, Krausz KW and Gonzalez FJ: Peroxisome proliferator-activated receptor alpha induction of uncoupling protein 2 protects against acetaminophen-induced liver toxicity. Hepatology 56: 281-290, 2012.

This work is licensed under a Creative Commons

Attribution-NonCommercial-NoDerivatives 4.0 International (CC BY-NC-ND 4.0) License. 